Check for updates

Cite this: Chem. Sci., 2019, 10, 4854

๑ All publication charges for this article have been paid for by the Royal Society of Chemistry

Received 17th December 2018

Accepted 25th March 2019

DOI: $10.1039 / \mathrm{c} 8 \mathrm{sc} 05613 \mathrm{~g}$

rsc.li/chemical-science

\section{Facile access to functionalized chiral secondary benzylic boronic esters via catalytic asymmetric hydroboration $\uparrow$}

\author{
Suman Chakrabarty, (D) ${ }^{a}$ Hector Palencia, (D) ${ }^{\mathrm{b}}$ Martha D. Morton, (D) ${ }^{\text {ac }}$ Ryan O. Carr (D) a \\ and James M. Takacs (D) *ac
}

Allylic and homoallylic phosphonates bearing an aryl or heteroaryl substituent at the $\gamma$ - or $\delta$-position undergo rhodium-catalyzed asymmetric hydroboration by pinacolborane to give functionalized chiral secondary benzylic boronic esters in yields up to $86 \%$ and enantiomer ratios up to $99: 1$. Compared to minimallyfunctionalized terminal and 1,1-disubstituted vinyl arenes, there are relatively few reports of efficient catalytic asymmetric hydroboration (CAHB) of more highly functionalized internal alkenes. Phosphonate substrates bearing a variety of common heterocyclic ring systems, including furan, indole, pyrrole and thiophene derivatives, as well as those bearing basic nitrogen substituents (e.g., morpholine and pyrazine) are tolerated, although donor substituents positioned in close proximity of the alkene can influence the course of the reaction. Stereoisomeric (E)- and (Z)-substrates afford the same major enantiomer of the borated product. Deuterium-labelling studies reveal that rapid $(Z)$ - to $(E)$-alkene isomerization accounts for the observed (E/Z)-stereoconvergence during CAHB. The synthetic utility of the chiral boronic ester products is illustrated by stereospecific $C-B$ bond transformations including stereoretentive electrophile promoted 1,2-B-to-C migrations, stereoinvertive $\mathrm{S}_{\mathrm{E}} 2$ reactions of boron-ate complexes with electrophiles, and stereoretentive palladium- and rhodium-catalyzed cross-coupling protocols.

\section{Introduction}

Chiral alkyl boronic esters possess a unique blend of benchtop stability and the potential to undergo a variety of C-B bond transformations via stereospecific 1,2-migration from an in situ generated boron "ate-complex" rendering them especially versatile intermediates for asymmetric synthesis. ${ }^{1,2}$ Metalcatalyzed asymmetric protoboration ${ }^{3}$ and hydroboration ${ }^{4,5}$ of alkenes are among the most common approaches for the preparation of chiral alkyl boronic esters. While catalytic asymmetric hydroboration (CAHB) of minimally-functionalized terminal and 1,1-disubstituted vinyl arenes (e.g. simple substituted styrene derivatives) have been extensively investigated, ${ }^{6}$ there are relatively few reports using more highly functionalized di- or trisubstituted internal alkenes. ${ }^{7}$ The latter have been a focus of our research into CAHB. Herein, we disclose that 1,2-disubstituted allyl phosphonates bearing an aryl substituent

\footnotetext{
${ }^{a}$ Department of Chemistry, University of Nebraska-Lincoln, Lincoln, Nebraska 68588-0304, USA. E-mail: jtakacs1@unl.edu

${ }^{b}$ Department of Chemistry, University of Nebraska-Kearney, Kearney, Nebraska 68849 , USA

${ }^{c}$ Nebraska Center for Integrated Biomolecular Communication (NCIBC), University of Nebraska-Lincoln, Lincoln, Nebraska 68588-0304, USA

$\dagger$ Electronic supplementary information (ESI) available: Experimental procedures and characterization data \& dpectral data. See DOI: 10.1039/c8sc05613g
}

at the $\gamma$-position are efficient substrates and provide facile access to functionalized chiral, secondary benzylic and related $\alpha$-aryl boronic esters. ${ }^{8}$

We previously reported that methylidene substrates bearing $\beta$-phenyl substitution undergo efficient rhodium-catalyzed CAHB with pinacolborane (pinBH) to give chiral tertiary boronic esters via regioselective $\beta$-boration. For example, 1 affords $(R)-2(81 \%)$ in a $97: 3$ enantiomer ratio (er); the $\beta: \gamma$ regioisomer ratio (rr) is $4: 1$ or greater under the conditions described in Fig. $1 .^{5 a}$ Similarly, the trisubstituted alkene $(E)-3$, bearing phenyl substituents at both the $\beta$ - and $\gamma$-positions, undergoes preferential $\beta$-boration ( $3: 1 \mathrm{rr}$ ) to afford the chiral, tertiary boronic ester product $(R)-4\left(60 \%, 97: 3\right.$ er). ${ }^{5 a}$

We now report that $\mathbf{5 a}$ and related substrates bearing a phenyl, aryl or heteroaryl substituent at the $\gamma$-position undergo preferential $\gamma$-boration to afford new chiral, secondary benzylic boronic esters. The benzylic regiochemistry presumably arises from the favorable formation of a rhodium $\pi$-benzyl complex of the substrate in the course of reaction. ${ }^{9}$ We were surprised to find that the alkene stereochemistry does not impact the overall regio- or stereochemical course of the reaction. Both $(E)$ - and $(Z)-5 \mathbf{a}$ afford the same $\gamma$-borated product $\mathbf{6 a}$ in greater than $20: 1 \mathrm{rr}$ using $(R)-\mathbf{B} \mathbf{1}$; $\mathrm{C}-\mathrm{B}$ bond oxidation using $\mathrm{NaBO}_{3} \cdot 4 \mathrm{H}_{2} \mathrm{O}$ affords the known chiral alcohol ${ }^{10}$ shown $(82 \%$, $96: 4$ er). 
Prior work: CAHB of $\beta$-aryl methylidenes $\& \beta, \gamma$-bisaryl trisubstituted alkenes

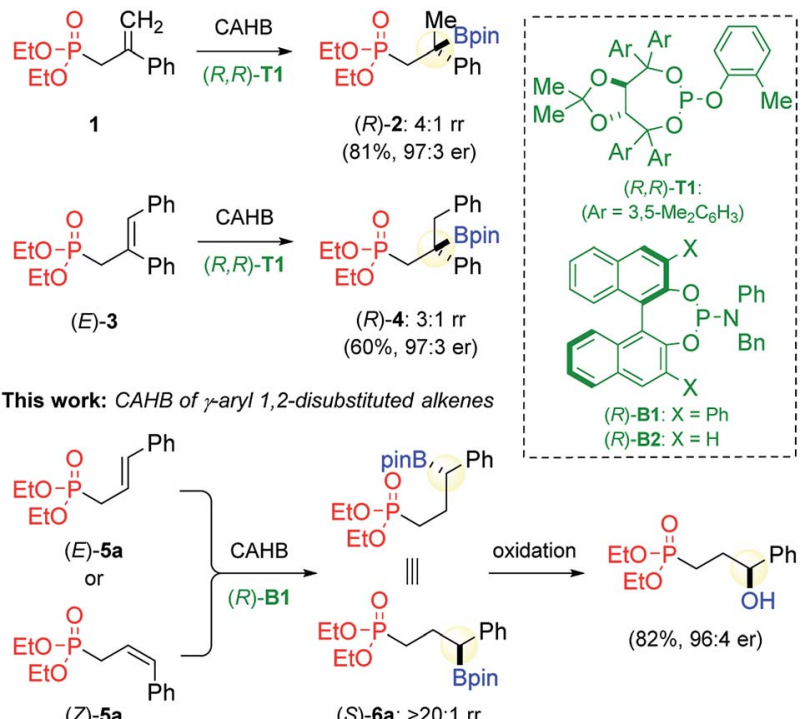

Fig. 1 Directed-CAHB of allylic phosphonate-functionalized vinyl arenes varying in substitution pattern. Standard CAHB conditions: $1 \mathrm{~mol} \% \mathrm{Rh}(\mathrm{nbd})_{2} \mathrm{BF}_{4}$ or $0.5 \mathrm{~mol} \%\left[\mathrm{Rh}(\mathrm{cod}) \mathrm{Cl}_{2} / 1 \mathrm{~mol} \% \mathrm{AgBF}_{4}, 1 \mathrm{~mol} \%\right.$ $(R, R)-\mathrm{T} 1$ or $(R)-\mathrm{B} 1,1.1$ eq. pinacolborane (pinBH), $3 \mathrm{~h}$, rt. Oxidation conditions: $\mathrm{NaBO}_{3} \cdot 4 \mathrm{H}_{2} \mathrm{O} / \mathrm{H}_{2} \mathrm{O}$

\section{Results and discussions}

Two alternative rhodium catalyst precursors are used in this study. A $1: 1$ combination of $\left[\mathrm{Rh}(\mathrm{nbd})_{2} \mathrm{BF}_{4} /(R)-\mathbf{B 1}\right]$ can be replaced by an in situ generated $1: 1\left[\mathrm{Rh}(\operatorname{cod}) \mathrm{BF}_{4} /(R)\right.$-B1] catalyst formed by treating $[\mathrm{Rh}(\operatorname{cod}) \mathrm{Cl}]_{2}$ with $\mathrm{AgBF}_{4}$ and the chiral ligand. The catalysts afford near identical results, but the latter protocol is more economical. While pinBH is known to undergo relatively facile rhodium-catalyzed degradation, ${ }^{11}$ thus often necessitating its use in excess, a stoichiometric amount of pinBH is usually enough for complete CAHB of these allylic phosphonates.

The choice of chiral ligand is, of course, critical to the success of CAHB. ${ }^{12}$ As indicated in Fig. 1, rhodium catalyst systems incorporating the TADDOL-derived chiral cyclic monophosphite $(R, R)$-T1 give efficient enantioinduction for $\beta$ aryl methylidene substrate $\mathbf{1}$ and the $\beta, \gamma$-bisaryl trisubstituted alkene $(E)$-3. Its use with 5a gives $(S)$-6a in good yield (81\%, after oxidation), but with only a low level of enantioinduction ( $64: 36$ er). In contrast, the BINOL-derived phosphoramidite $(R)$-B1 affords high levels of regiocontrol and enantioselectivity. ${ }^{13}$

\section{Scope of aryl and heteroaryl substrates}

Fig. 2 summarizes results obtained for a series of substrates $\mathbf{5 b} \mathbf{b}$ $\mathbf{t}$ in which the nature of the aromatic ring appended at the $\gamma$ position varies. In addition to the major $\gamma$-boration product 6 and the minor regioisomeric $\beta$-boration product 7 , alkene reduction (i.e., 8) typically comprises the remaining $5-15 \%$ of the product mixture. The latter reaction mode arises from a competing catalytic cycle and is the subject of ongoing studies $;{ }^{14}$ it will not be discussed further here. Herein, we focus on the regio- and enantioselectivity of the rhodium-catalyzed hydroboration mode. ${ }^{15}$

A range of donor and acceptor substituents are well-tolerated with relatively minor fluctuations in regio- and/or enantioselectivity. For example, the 4-methylphenyl derivative $\mathbf{5 b}$ and the 4-trifluoromethylphenyl derivative 5c undergo $\gamma$-boration with high regioselectivity ( $>20: 1 \mathrm{rr})$ yielding $9 \mathrm{~b}(84 \%, 97: 3 \mathrm{er})$ and 9c $(78 \%, 97: 3$ er) after oxidation of the corresponding secondary benzylic boronic esters (i.e., $\mathbf{6 b}$ and $\mathbf{6 c}$ ). The 4 -fluorophenyl (5d) and 4-methyoxyphenyl (5e) derivatives exhibit somewhat lower levels of $\gamma: \beta$ regioselectivity (6d-e, 10-11:1 rr) and enantioselectivity affording $\mathbf{9 d}(71 \%, 94: 6 \mathrm{er})$ and $\mathbf{9 e}$ (77\%, $94: 6$ er), respectively. In contrast, the 3-methoxyphenyl derivative $\mathbf{5 f}$ and 3,4-methylenedioxyphenyl derivative $\mathbf{5 g}$ again exhibit higher regioselectivity (6f-g: $>20: 1 \mathrm{rr}$ ) leading to 9 f (83\%, $98: 2$ er) and $9 \mathrm{~g}(70 \%, 94: 6 \mathrm{er})$, after oxidation. The 2 methoxyphenyl derivative $5 \mathbf{h}$ exhibits reduced regioselectivity (6h: $9: 1 \mathrm{rr}$ ), but otherwise good conversion to yield $9 \mathbf{h}$ ( $72 \%$, $94: 6$ er). ${ }^{16}$ The 4 -dimethylamino, morpholine, and pyrazine derivatives $\mathbf{5 i}-\mathbf{k}$ demonstrate the viability of substrates bearing basic nitrogen functionality; $\mathbf{9 i - k}$ are obtained in moderate to good yields (55-68\%) and up to $94: 6$ er.

In addition to demonstrating tolerance for basic nitrogen in several of the substrates described in Fig. 2, it is pleasing to see that substrates incorporating some heteroaromatic ring systems also undergo efficient CAHB, albeit with some unusual variation in regio- and/or enantioselectivity. For example, the Boc-protected indole derivative $\mathbf{5 l}$ exhibits high $\gamma$-regioselectivity (61, >20:1 rr) with good enantioinduction after oxidation to 91 (78\%, $96: 4$ er). Similarly, the 3-substituted $N$-Bocprotected pyrrole derivative $\mathbf{5 m}$ is both highly regioselective (6m, >20:1 rr) and highly enantioselective; the er obtained for 9m $(86 \%, 99: 1$ er $)$ is the highest obtained among the substrates tested. However, the results obtained for CAHB of the 2-substituted $N$-Boc-protected pyrrole derivative $5 n$ differ markedly. The regioselectivity of $\mathbf{6 n}(>20: 1 \mathrm{rr})$ is excellent. However, the level of enantioselectivity for $9 \mathbf{n}(85 \%, 80: 20 \mathrm{er})$ is not only much lower but results from hydroboration with the opposite sense of $\pi$-facial selectivity compared to most other substrates (vide infra); $(R)-9 n$ is the major product.

The 3- and 2-substituted thiophene substrates (i.e., 50 and 5p) exhibit high regioselectivity leading to 60 and $6 p$ (>20:1 rr), respectively. However, the 3 -substituted thiophene $\mathbf{5 0}$ affords $\mathbf{9 0}$ $(83 \%, 85: 15 \mathrm{er})$ with only modest levels of stereocontrol, while the 2 -substituted thiophene $5 \mathbf{p}$ gives $\mathbf{9 p}(80 \%, 95: 5$ er) with good stereocontrol. The 2 -substituted benzothiophene $\mathbf{5 q}$ gives both lower levels of regiocontrol (6q, $5: 1 \mathrm{rr}$ ) and enantioselectivity for 9q $(65 \%, 89: 11 \mathrm{er})$. The corresponding furan derivatives $\mathbf{5 r}-\mathbf{t}$ give similar results.

\section{Stereochemical assignments}

The variable regio- and stereochemical results obtained with heteroaryl derivatives illustrate a caveat for substrates bearing multiple donor groups in proximity to the alkene or bear relatively bulky aromatic ring systems. Using the Birman 

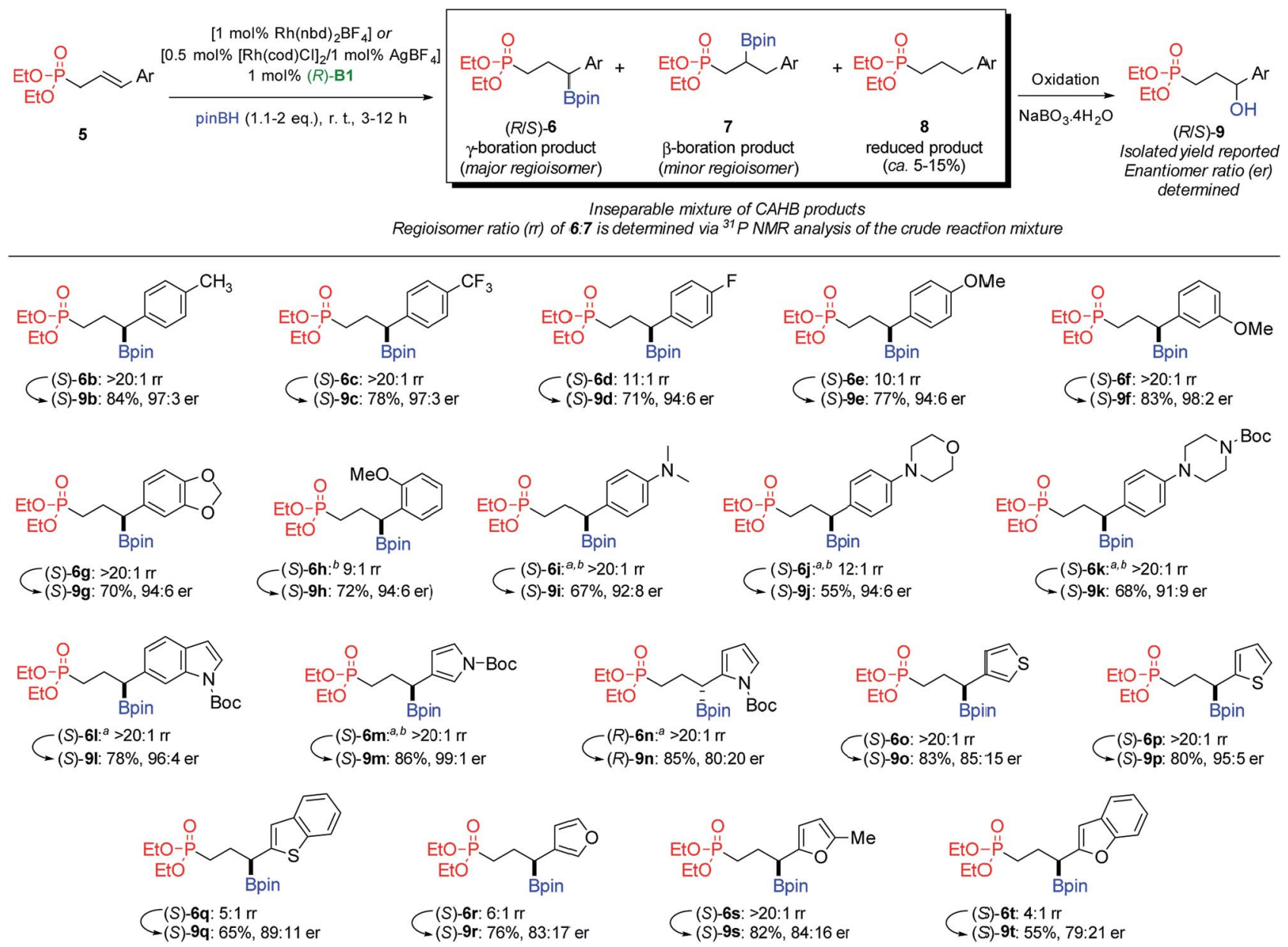

Fig. 2 Substrate scope for CAHB of $\gamma$-aryl allylic phosphonates. Note: ${ }^{\text {atwo }}$ equivalents of pinacolborane were used. ${ }^{b}$ Reaction carried out for 12 hours.

benzotetramisole $(\mathrm{BTM})^{17}$ chiral acylation catalyst, a kinetic resolution (KR) strategy was employed to confirm the absolute configuration assignments for several of the previously unreported chiral secondary benzylic alcohols 9 prepared via CAHB. As shown in Fig. 3A, the (S)-BTM-catalyzed acylation of a racemic sample of $( \pm)$-9a with 0.5 equivalents of isobutyric anhydride results in the rapid acylation of $(S)-\mathbf{9 a}$ to $(S)$-10a and recovery of the known $(R)$-9a unreacted alcohol.

Alcohol 9 and ester 10 are readily differentiated by ${ }^{31} \mathrm{P}$ NMR spectroscopy providing a convenient protocol for the rapid determination of absolute configuration of these previously unreported chemical entities (Fig. 3B). As demonstrated by the ${ }^{31}$ P NMR stack-plot, $(S)$-9a (96: 4 er) is more rapidly acylated using ( $S$ )-BTM than with (R)-BTM. Using this method, we assigned the absolute configurations for the $\alpha$-hydroxy heteroaryl products obtained via $\mathrm{CAHB} /$ oxidation (Fig. 3C). For example, the 3-substituted pyrrole derivative 9m (99:1 er), synthesized via $\mathrm{CAHB} /$ oxidation using $(R)$-B1, undergoes more rapid (S)-BTM-catalyzed acylation to $\mathbf{1 0 m}(27 \%$ conversion in 12 h) compared to $(R)$-BTM-catalyzed acylation ( $2 \%$ conversion in $12 \mathrm{~h})$. The relative rates are consistent with predominant $(S)$ configuration of $9 \mathrm{~m}$. In contrast, the 2-pyrrole derivative $9 \mathbf{n}$
(80 : 20 er), also prepared via CAHB/oxidation, undergoes relatively sluggish $(S)$-BTM-catalyzed acylation to 10n compared to $(R)$-BTM-catalyzed acylation. The results indicate that predominantly $(R)-9 \mathbf{n}$ is formed from the 2-substituted pyrrole by CAHB with $(R)$-B1. We speculate that the $N$-Boc moiety in the latter acts as an alternative directing group in the rhodium-catalyzed hydroboration thereby switching the sense of alkene $\pi$-facial selectivity. Although one might reasonably expect that 2 -subsituted thiophene and furan derivatives behave similarly, the data summarized in Fig. $3 \mathrm{c}$ indicate that $(S)$-9o-p and $(S)-\mathbf{9 r}-\mathbf{s}$ are the major stereoisomers produced via CAHB/oxidation.

In our prior studies of phosphonate-directed CAHB, we illustrated the synthetic utility of chiral bifunctional tertiary organoboron derivatives through thiophosphonate olefination $^{5 b}$ chemistry and $\alpha$-oxophosphonate active ester ${ }^{5 a}$ chemistry as well as through a number of stereospecific C-B bond transformations. Here, we focus on the use of $(S)-6 \mathbf{a}(96: 4 \mathrm{er})$ in stereoretentive and stereoinvertive $\mathrm{C}-\mathrm{B}$ bond transformations via intermediate boron-ate complexes (Fig. 4). Stereoretentive cross-coupling with carbanions derived from electron rich vinyl and aromatic derivatives via electrophile-promoted 1,2-B-to-C migration of a boron-ate complex is generally facile under 
A. General Scheme for kinetic resolution via acylation using benzotetramisole (BTM)

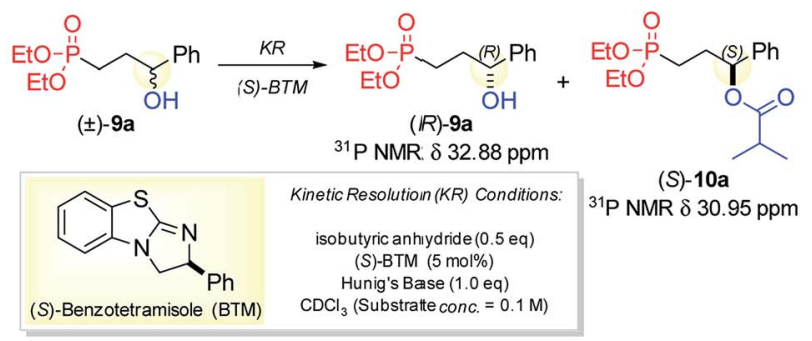

B. ${ }^{31}$ P NMR analysis for assignment of absolute configuration via $K R$

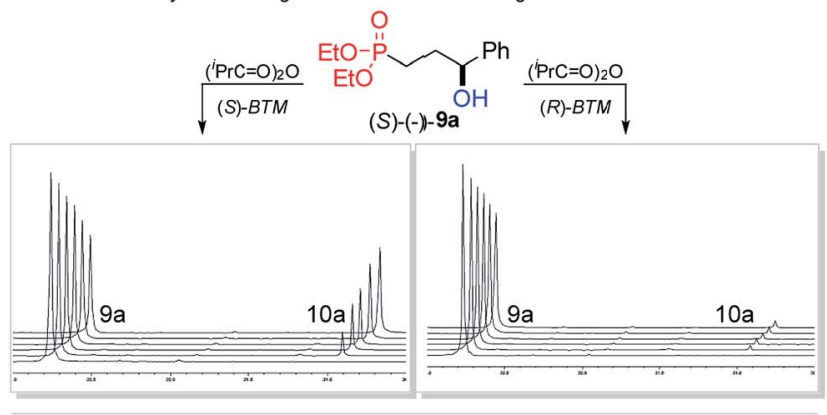

C. Absolute configuration assignments of benzyl alcohol products containing heterocyclic ring systems via KR

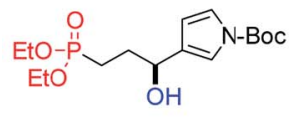

(S)-9m (99:1 er) (R)-BTM: $2 \%$ conv. $(12 \mathrm{~h})$ (S)-BTM: $27 \%$ conv. $(12 \mathrm{~h})$

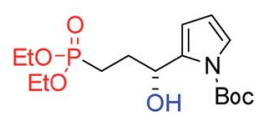

(R)-9n (80:20 er)

(R)-BTM: $21 \%$ conv. $(12 \mathrm{~h})$

(S)-BTM: $3 \%$ conv. (12 hi

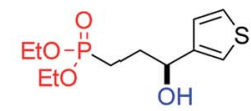

(S)-9o (85:15 er) (R)-BTM: $4 \%$ conv. (1 h) (S)-BTM: $30 \%$ conv. ( $1 \mathrm{~h})$

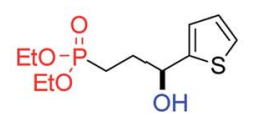

(S)-9p (95:5 er)

(R)-BTM: $2 \%$ conv. $(1 \mathrm{~h})$ (S)-BTM: $31 \%$ conv. (1 h)

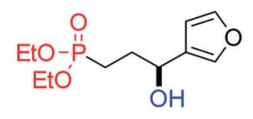

(S)-9r (83:17 er) (R)-BTM: $6 \%$ conv. (12 h) (S)-BTM: $41 \%$ conv. (12 h)

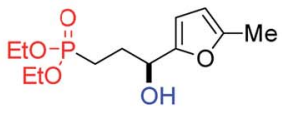

(S)-9s (84:16 er) (R)-BTM: $3 \%$ conv. ( $12 \mathrm{~h}$ ) (S)-BTM: $14 \%$ conv. (12 h)
Fig. 3 Stereochemical assignments based on kinetic resolution: (A) General scheme for kinetic resolution via acylation using benzotetramisole (BTM); (B) ${ }^{31} \mathrm{P}$ NMR analysis for assignment of absolute configuration via $\mathrm{KR}$; (C) absolute configuration assignments of benzyl alcohol products containing heterocyclic ring systems via KR.

conditions reported by Aggarwal. ${ }^{2 \boldsymbol{b}, 2 e}$ For example, treatment of $(S)$-6a with excess vinyl magnesium bromide followed by $\mathrm{I}_{2}$ and sodium methoxide affords the vinyl derivative $(R)-\mathbf{1 1}$ (79\%, $95: 5 \mathrm{er})$ in high levels of stereoretention. ${ }^{2 e}$ Similarly, reaction of $(S)$-6a with 2-lithiobenzofuran followed by NBS affords the gem-bisaryl product $(S)-\mathbf{1 2}(68 \%, 96: 4$ er) with essentially complete enantiospecificity. ${ }^{2 b}$

The palladium-catalyzed cross-coupling of chiral boronic esters has attracted recent interest both due to its synthetic utility and the interesting mechanistic issue of stereoretention ${ }^{\mathbf{1 8}}$ or stereoinversion; ${ }^{19}$ the outcome is often dependent upon participation or non-participation of polar substituents in the substrate. $^{\mathbf{2 0 , 2 1}}$ Benzylic boronic esters are rather unique in the context of palladium-catalyzed cross-coupling. Only the protocol recently introduced by Crudden ${ }^{22}$ is reportedly effective. Its efficiency varies, but in favorable constructs, the latter proceeds with $84-94 \%$ stereoretention (sr). Thus, the outcome

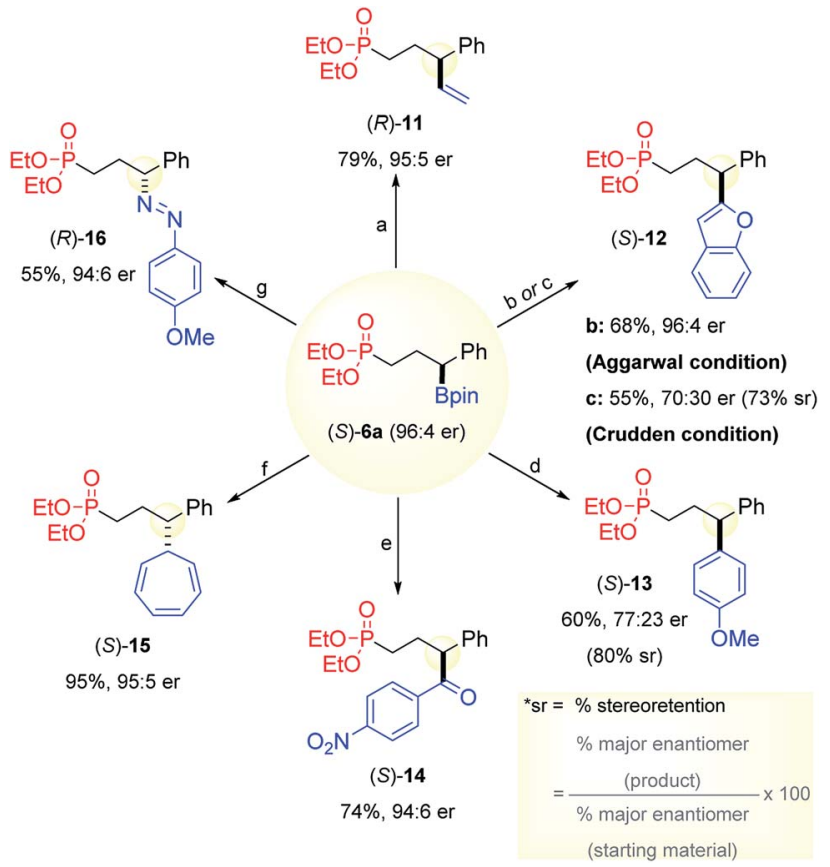

Fig. 4 Product utility illustrated by stereoretentive and stereoinvertive $\mathrm{C}-\mathrm{B}$ bond transformations. Reagents and conditions: (a) (i) excess $\mathrm{CH}_{2}=\mathrm{CHMgBr}$, $\mathrm{THF},-78{ }^{\circ} \mathrm{C}$ to rt; (ii) $\mathrm{I}_{2} / \mathrm{MeOH},-78{ }^{\circ} \mathrm{C}$; (iii) $\mathrm{NaOMe} /$ $\mathrm{MeOH}$; (iv) $\mathrm{Na}_{2} \mathrm{~S}_{2} \mathrm{O}_{3}$ (aq.); (b) (i) benzofuran-2-yllithium, $\mathrm{THF},-78^{\circ} \mathrm{C}$; (ii) NBS, THF, $-78{ }^{\circ} \mathrm{C}$; (iii) $\mathrm{Na}_{2} \mathrm{~S}_{2} \mathrm{SO}_{3}$ (aq.); (c) 2-iodobenzofuran, $\mathrm{Ag}_{2} \mathrm{O}$, $\mathrm{Pd}_{2}(\mathrm{dba})_{3} / \mathrm{PPh}_{3}, \mathrm{THF}, 6{ }^{\circ} \mathrm{C}$; (d) 4-iodoanisole, $\mathrm{Ag}_{2} \mathrm{O}, \mathrm{Pd}_{2}(\mathrm{dba})_{3} / \mathrm{PPh}_{3}$, $\mathrm{THF}, 60{ }^{\circ} \mathrm{C}$; (e) (i) 4-nitrobenzaldehyde, $\left[\mathrm{Rh}(\operatorname{cod}) \mathrm{Cl}_{2}, \mathrm{KHF}_{2}\right.$, dioxane/ $\mathrm{H}_{2} \mathrm{O}$; (ii) TEMPO, TCCA, $\mathrm{CH}_{2} \mathrm{Cl}_{2}$; (f) (3,5-bis(trifluoromethyl)phenyl) lithium, THF, $-78{ }^{\circ} \mathrm{C}$ to $-40{ }^{\circ} \mathrm{C}$; (ii) cycloheptatrienyl tetrafluoroborate, rt.; (iii) $\mathrm{NaHCO}_{3}$ (aq.). (g) (i) (3,5-bis(trifluoromethyl) phenyl)lithium, THF, $-78{ }^{\circ} \mathrm{C}$ to $-40{ }^{\circ} \mathrm{C}$; (ii) 4-Methoxybenzenediazonium tetrafluoroborate, $0{ }^{\circ} \mathrm{C}$ (iii) $\mathrm{NaHCO}_{3}$ (aq.).

for the bifunctional substrate $(S)-6 \mathbf{a}$ (96 : 4 er) was uncertain. In our hands, cross-coupling with 2-iodobenzofuran under the $\left[\mathrm{Pd}_{2}(\mathrm{dba})_{3} / \mathrm{PPh}_{3} / \mathrm{Ag}_{2} \mathrm{O}\right]$ conditions reported by Crudden gives $(S)-12$ (55\%, 70 : $30 \mathrm{er}, 73 \% \mathrm{sr})$; cross-coupling proceeds with predominant stereoretention, albeit with significant erosion of enantiopurity. Using the same conditions, cross-coupling of $(S)$ 6a with 4-iodoanisole yields $(S)-\mathbf{1 3}$ (60\%, 77 : 23 er, 80\% sr). The latter is useful, since we were unable to prepare 13 via the 1,2-Bto-C migration protocol. An alternative rhodium-catalyzed cross-coupling procedure reported by Aggarwal $^{23}$ effects the stereoretentive addition of $(S)$-6a to 4-nitrobenzaldehyde to afford aryl ketone $(S)-\mathbf{1 4}(74 \%, 94: 6$ er) after oxidation; the latter cross-coupling proceeds with only slight erosion of enantiopurity over two steps.

Aggarwal reported that ate-complexes of secondary boronic esters react with strong electrophiles via a stereoinvertive $\mathrm{S}_{\mathrm{E}} 2$ mechanism. ${ }^{2 f}$ We find that the intermediate boron-ate complex formed by addition of (3,5-bis(trifluoromethyl)phenyl) lithium to $(S)-6 \mathbf{a}(96: 4$ er) readily reacts with cycloheptatrienyl tetrafluoroborate to effect the net $\mathrm{C}-\mathrm{B}$ to $\mathrm{C}-\mathrm{C}$ bond substitution to give $(S)-15$ (95\%, 95 : 5 er) in excellent yield. Similarly, the net stereoinvertive $\mathrm{C}-\mathrm{B}$ to $\mathrm{C}-\mathrm{N}$ bond substitution is accomplished by treating the in situ generated boron ate-complex with 4- 
A. Analogous amide substrate undergoes efficient $C A H B$ using B1<smiles>O=C(CC=Cc1ccccc1)NCc1ccccc1</smiles>

$(E)-17$

(S)-18: 7:1 rr (78\%, 94:6 er)

B. One-carbon homologous substrates perform with similar efficiency: Same major enantiomer obtained from (E)- and (Z)-substrates

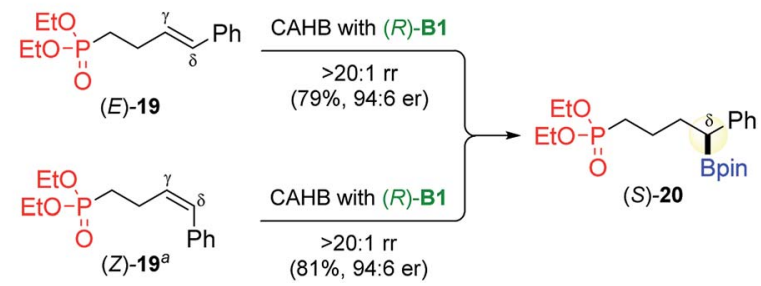

Fig. 5 The influence of other structural variations on the directed CAHB of 1,2-disubstituted alkenes: (A) analogous amide substrate undergoes efficient CAHB using B1; (B) one-carbon homologous substrates perform with similar efficiency: Same major enantiomer obtained from $(E)$ - and (Z)-substrates. Note: Yield and er are determined after oxidation to the corresponding alcohols. ${ }^{\mathrm{a}} Z: E$ ratio $=4: 1$.

methoxybenzenediazonium tetrafluoroborate to give the diazo compound $(R)-16$ (55\%, $94: 6$ er).

\section{Additional substrate scope and some key mechanistic insights}

The question naturally arises as to whether the phosphonate directing group is unique in promoting $\gamma$-boration with these vinyl arene substrates. That appears not to be the case (Fig. 5A); the corresponding benzylamide substrate $(E)-\mathbf{1 7}$ undergoes regioselective (7 : $1 \mathrm{rr}$ ) amide-directed CAHB using $(R)$-B1 to afford the analogous $\gamma$-borated benzylic ester $(S)$-18 (78\%, $94: 6$ er). ${ }^{24}$

The one-carbon homolog of $(E)-5 \mathbf{a}$, that is, the $\gamma, \delta$-unsaturated phosphonate substrate $(E)-\mathbf{1 9}$, reacts under the standard conditions to afford predominantly (>20:1 rr) $\delta$-borated benzylic boronic ester $(S)-20(79 \%, 94: 6$ er) after oxidation (Fig. 5B). Furthermore, as was noted for $(E)$ - and $(Z)-5 \mathbf{a}$, the same major enantiomer of $\mathbf{2 0}$ is formed independent of the substrate alkene geometry; $(Z)-19$ also affords $(S)-20$ (81\%, $94: 6$ er). The ability to start with pure $(E)$-, pure $(Z)$ - or an $(E / Z)$-mixture and arrive at the same product is a practical advantage but raises mechanistic questions. We previously reported that the amidedirected CAHBs of $(E)$ - and $(Z)$-trisubstituted alkenes proceed with the same sense of $\pi$-facial selectivity and therefore lead to diastereomers. ${ }^{5 f}$ Those results would have suggested that $(E)$ and (Z)-19 (and similarly (E)- and (Z)-5a) should lead to enantiomers not the same product.

The origin of $(E / Z)$-isomer stereoconvergence is resolved based on the results of CAHB of $(Z)$-5a using a limiting amount of pinBH (Fig. 6A) and deuterium labelling via CAHB of $(E)$ - and (Z)-5a with pinBD (Fig. 6B). With respect to the first test, a sample enriched in $(Z)$-isomer of $5 \mathbf{a}(90: 10 Z: E)$ is subjected to the otherwise standard CAHB conditions, but using a limiting amount of pinBH ( 0.4 equiv.), leading to partial boration and recovered alkene. The ${ }^{1} \mathrm{H}$ NMR spectral windows for the starting and recovered $E / Z$-mixtures of 5 a shown in
A. (Z)- to (E)-substrate isomerization is observed under standard CAHB conditions:
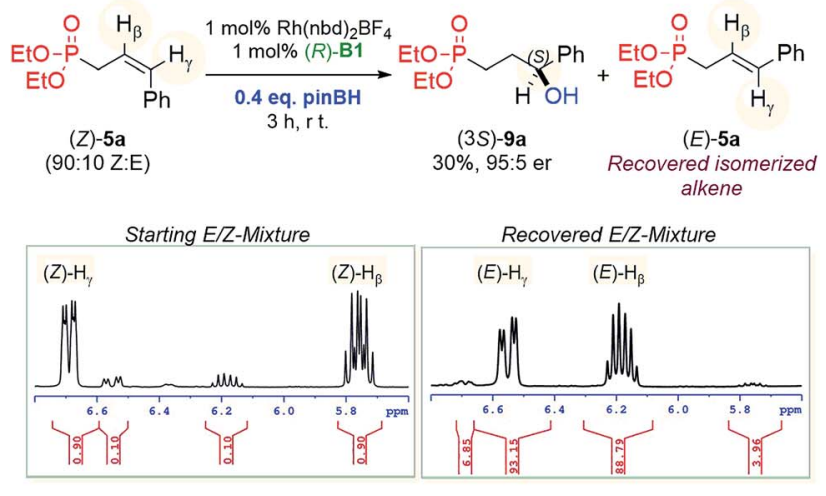

B. Different distributions of deuterated products are obtained when isomeric (E)- or (Z)-substrates react with pinBD under standard $C A H B$ conditions:

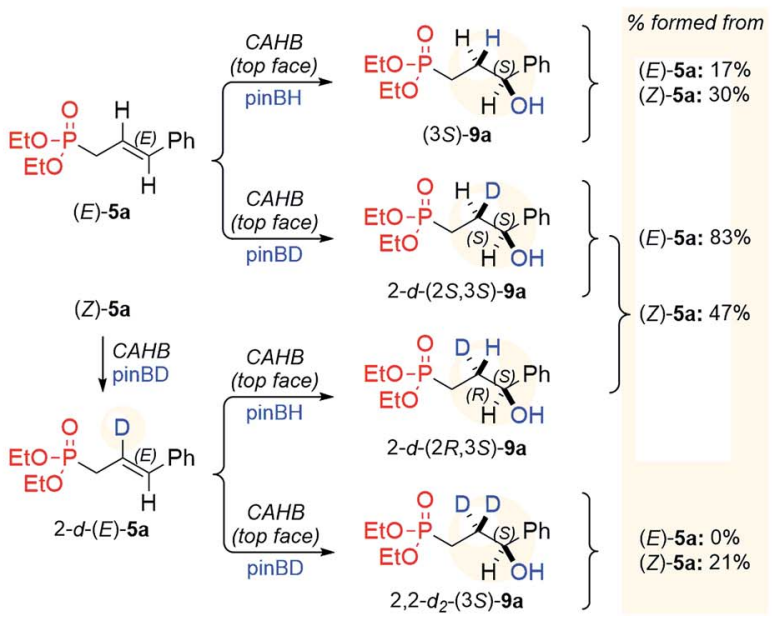

Fig. 6 Mechanistic studies indicating the origin of $(E / Z)$-stereoconvergent $\mathrm{CAHB}$ arising via $Z$ to $E$ isomerization under the reaction conditions: $(A)(Z)$ - to $(E)$-substrate isomerization is observed under standard CAHB conditions; (B) different distributions of deuterated products are obtained when isomeric $(E)$ - or $(Z)$ - substrates react with pinBD under standard CAHB conditions.

Fig. 6A indicate that $(Z)-5 a$ is essentially completely converted to the $(E)$-isomer under the reaction conditions, thus providing a mechanism by which the two isomers lead to the same product.

Fig. 6B shows the results of deuterium labelling. CAHB of $(E)$ and $(Z)-5 a$ using pinBD affords different distributions of non-, isomeric mono- and di-deuterated products. CAHB/oxidation of

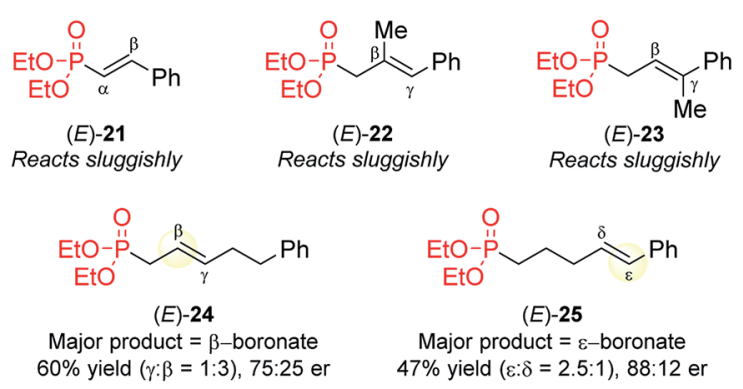

Fig. 7 Current limitations of the methodology. 
(E)-5a using pinBD yields essentially a single monodeuterated product $2-d-(2 S, 3 S)-9 \mathrm{a} \quad(83 \%)$ accompanied by the nondeuterated $(3 S)-9 \mathbf{9}(17 \%)$ with no apparent di-deuteration as determined by mass spectral analysis. In contrast, CAHB/ oxidation of (Z)-5a using pinBD affords: $30 \%$ of nondeuterated $(3 S)-\mathbf{9 a} ; 47 \%$ of a mixture of diastereomeric monodeuterated products $2-d-(2 S, 3 S)-\mathbf{9 a}$; and $2-d-(2 R, 3 S)-\mathbf{9 a}$ and $21 \%$ of the dideuterated product $2,2-d_{2}-(3 S)-9 \mathbf{a}$. The dideuterated product presumably arises by the reaction of $2-d-(E)-5 \mathbf{a}$ with pinBD. The latter, when generated in situ via isomerization of (Z)-5a as described above, also generates an equivalent amount of pinBH that can react competitively to give the observed diastereomeric monodeuterated product and an increased amount of the non-deuterated product as is observed. See the ESI $\uparrow$ for more detailed mechanistic schemes.

\section{Some current limitations}

The attempted CAHB of several related phosphonates reveal some current limitations of the $\left[\mathrm{Rh}(\mathrm{nbd})_{2} \mathrm{BF}_{4} / \mathbf{B 1}\right]$ catalyst system (Fig. 7). (i) The vinyl (i.e., $\alpha, \beta$-unsaturated) phosphonate $(E)-21$ is largely recovered unchanged when subjected to the standard CAHB conditions. (ii) The trisubstituted variants $(E)$ 22 and $(E)-23$ react only sluggishly under the standard conditions. (iii) In contrast to the vinylarene substrate $(E)-5 \mathbf{a}$, similar internal alkenes bearing an alkyl rather than aryl/heteroaryl $\gamma$ substituent (e.g., $(E)$-24), undergo predominantly $\beta$-boration, albeit with modest regio- and enantioselectivity with the standard catalyst system. (iv) The $\delta, \varepsilon$-unsaturated vinyl arene $(E)$-25 affords the corresponding benzylic boronic ester upon CAHB but again with only modest regioselectivity, yield, and enantiopurity. It should be noted, however, that these results can only be said to reflect limitations of the $\left[\mathrm{Rh}(\mathrm{nbd})_{2} \mathrm{BF}_{4} / \mathbf{B} \mathbf{1}\right]$ catalyst system; systematic catalyst optimizations have not been carried out for these substrates.

\section{Conclusions}

While the regioselective, CAHBs of minimally-functionalized terminal and 1,1-disubstituted vinyl arenes (e.g. simple substituted styrene derivatives) have been investigated rather extensively and quite successfully, there are relatively few reports using more highly functionalized di- or trisubstituted internal alkenes. We find that the rhodium-catalyzed CAHBs of allylic and homoallylic phosphonates derived from internal vinyl arenes give facile access to functionalized chiral secondary benzylic boronic esters. A range of substrates including some bearing heteroaromatic ring systems of interest in medicinal chemistry, such as furan, indole, morpholine, pyrazine, pyrrole and thiophene derivatives, can be accommodated. The absolute configurations of selected chiral secondary benzylic boronic ester products were confirmed or assigned via kinetic acylation of the corresponding benzylic alcohols using the BTM acylation catalyst highlighting the potential for other donor substituents in proximity of the alkene to influence the stereochemical course of the reaction. Stereoretentive and stereoinvertive C-B bond transformation protocols highlight the versatility of this methodology. We find that palladium-catalyzed cross-coupling under the conditions reported by Crudden proceeds mostly with stereoretention. Diastereomeric substrates, for example, $(E)$ - and $(Z)$-5a and $(E)$ - and $(Z)-\mathbf{1 6}$, give the same borated product stereochemistry. Mechanistic studies reveal that $(Z)$ - to $(E)$ alkene isomerization occurs rapidly under the reaction conditions. This observation along with deuterium incorporation data provides a reasonable explanation for the origin of $(E / Z)$ stereoconvergence during CAHB.

\section{Conflicts of interest}

The authors declare no conflicts of interest.

\section{Acknowledgements}

Funding from the NIH National Institutes of General Medical Sciences (R01 GM100101) is gratefully acknowledged. We thank Dr Jian Zhang, Dr Jingshi Lu and Mr Nicholas Armada for assistance in preparing (Z)-5a and (Z)-19 via photoisomerization. ${ }^{25}$

\section{Notes and references}

1 For leading examples, see (a) J. Wu, P. Lorenzo, S. Zhong, M. Ali, C. P. Butts, E. L. Myers and V. K. Aggarwal, Nature, 2017, 547, 436; (b) B. S. L. Collins, C. M. Wilson, E. L. Myers and V. K. Aggarwal, Angew. Chem., Int. Ed., 2017, 56, 11700; (c) J. Schmidt, J. Choi, A. T. Liu, M. Slusarczyk and G. C. Fu, Science, 2016, 354, 1265; (d) C. M. Crudden, C. Ziebenhaus, J. P. G. Rygus, K. Ghozati, P. J. Unsworth, M. Nambo, S. Voth, M. Hutchinson, V. S. Laberge, Y. Maekawa and D. Imao, Nat. Commun., 2016, 7, 11065; (e) S. C. Matthew, B. W. Glasspoole, P. Eisenberger and C. M. Crudden, J. Am. Chem. Soc., 2014, 136, 5828.

2 For selected examples, see: (a) L. Zhang, G. J. Lovinger, E. K. Edelstein, A. A. Szymaniak, M. P. Chierchia and J. P. Morken, Science, 2016, 351, 70; (b) A. Bonet, M. Odachowski, D. Leonori, S. Essafi and V. K. Aggarwal, Nat. Chem., 2014, 6, 584; (c) M. Burns, S. Essafi, J. R. Bame, S. P. Bull, M. P. Webster, S. Balieu, J. W. Dale, C. P. Butts, J. N. Harvey and V. K. Aggarwal, Nature, 2014, 513, 183; (d) C. G. Watson, A. Balanta, T. G. Elford, S. Essafi, J. N. Harvey and V. K. Aggarwal, J. Am. Chem. Soc., 2014, 136, 17370; (e) R. P. Sonawane, V. Jheengut, C. Rabalakos, R. Larouche-Gauthier, H. K. Scott and V. K. Aggarwal, Angew. Chem., Int. Ed., 2011, 50, 3760; $(f)$ R. LaroucheGauthier, T. G. Elford and V. K. Aggarwal, J. Am. Chem. Soc., 2011, 133, 16794. For reviews, see: $(g)$ C. Sandford and V. K. Aggarwal, Chem. Commun., 2017, 53, 5481; (h) R. J. Armstrong and V. K. Aggarwal, Synthesis, 2017, 49, 3323; (i) D. Leonori and V. K. Aggarwal, Angew. Chem., Int. Ed., 2015, 54, 1082.

3 For key examples, see (a) B. Liu, H.-H. Wu and J. Zhang, ACS Catal., 2018, 8, 8318; (b) Y. Cai, X.-T. Yang, S.-Q. Zhang, F. Li, Y.-Q. Li, L.-X. Ruan, X. Hong and S.-L. Shi, Angew. Chem., Int. 
Ed., 2018, 57, 1376; (c) L. Chen, X. Zou, H. Zhao and S. Xu, Org. Lett., 2017, 19, 3676; (d) C. Jarava-Barrera, A. Parra, A. Lopez, F. Cruz-Acosta, D. Collado-Sanz, D. J. Cardenas and M. Tortosa, ACS Catal., 2016, 6, 442; (e) H. Lee, B. Y. Lee and J. Yun, Org. Lett., 2015, 17, 764; (f) Z. T. He, Y. S. Zhao, P. Tian, C. C. Wang, H. Q. Dong and G. Q. Lin, Org. Lett., 2014, 16, 1426; $(g)$ A. Parra, L. Amenos, M. Guisan-Ceinos, A. Lopez, J. L. G. Ruano and M. Tortosa, J. Am. Chem. Soc., 2014, 136, 15833; $(h)$ J. C. H. Lee, R. McDonald and D. G. Hall, Nat. Chem., 2011, 3, 894; (i) Y. Sasaki, C. Zhong, M. Sawamura and H. Ito, J. Am. Chem. Soc., 2010, 132, 1226; (j) Y. Lee and A. H. Hoveyda, J. Am. Chem. Soc., 2009, 131, 3160; (k) J. M. O'Brien, K. S. Lee and A. H. Hoveyda, J. Am. Chem. Soc., 2010, 132, 10630; (l) I.-H. Chen, L. Yin, W. Itano, M. Kanai and M. Shibasaki, J. Am. Chem. Soc., 2009, 131, 11664.

4 For selected examples, see: (a) W. J. Jang, S. M. Song, J. H. Moon, J. Y. Lee and J. Yun, J. Am. Chem. Soc., 2017, 139, 13660; (b) J. R. Smith, B. S. L. Collins, M. J. Hesse, M. A. Graham, E. L. Myers and V. K. Aggarwal, J. Am. Chem. Soc., 2017, 139, 9148; (c) Y. Xi and J. F. Hartwig, J. Am. Chem. Soc., 2016, 138, 6703; (d) N. Hu, G. Zhao, Y. Zhang, X. Liu, G. Li and W. Tang, J. Am. Chem. Soc., 2015, 137, 6746. 5 (a) S. Chakrabarty and J. M. Takacs, ACS Catal., 2018, 8, 10530; (b) S. Chakrabarty and J. M. Takacs, J. Am. Chem. Soc., 2017, 139, 6066; (c) G. L. Hoang and J. M. Takacs, Chem. Sci., 2017, 8, 4511; (d) V. M. Shoba, N. C. Thacker, A. J. Bochat and J. M. Takacs, Angew. Chem., Int. Ed., 2016, 55, 1465; (e) G. L. Hoang, Z. D. Yang, S. M. Smith, R. Pal, J. L. Miska, D. E. Perez, L. S. W. Pelter, X. C. Zeng and J. M. Takacs, Org. Lett., 2015, 17, 940; (f) S. M. Smith and J. M. Takacs, J. Am. Chem. Soc., 2010, 132, 1740; $(g)$ S. M. Smith, N. C. Thacker and J. M. Takacs, J. Am. Chem. Soc., 2008, 130, 3734.

6 Selected examples of CAHB of 1,1-disubstituted vinyl arenes: (a) Z. Wang, Z. He, R. Zhang, G. Zhang, G. Xu, Q. Zhang, T. Xiong and Q. Zhang, Org. Lett., 2017, 19, 3067; (b) H. Zhang and Z. Lu, ACS Catal., 2016, 6, 6596; (c) L. Zhang, Z. Zuo, X. Wan and Z. J. Huang, J. Am. Chem. Soc., 2014, 136, 15501; (d) R. Corberan, N. W. Mszar and A. H. Hoveyda, Angew. Chem., Int. Ed., 2011, 50, 7079; (e) C. Mazet and D. Gerard, Chem. Commun., 2011, 47, 298.

7 For CAHB of 1,2-disubstituted vinyl arenes, see: (a) G. L. Hoang, S. Zhang and J. M. Takacs, Chem. Commun., 2018, 54, 4838; (b) D. Noh, S. K. Yoon, J. Won, J. Y. Lee and J. Yun, Chem.-Asian J., 2011, 6, 1967. For CAHB of trisubstituted internal alkenes (bearing alkyl/aryl substituents), see ref. $5 a, b, d$ and $f$.

8 Other recent methods for synthesizing chiral secondary benzylic/ $\alpha$-aryl boronic esters: (a) Z. Tao, K. A. Robb, J. L. Panger and S. E. Denmark, J. Am. Chem. Soc., 2018, 140, 15621; (b) J. Guo, B. Cheng, X. Shen and Z. Lu, J. Am. Chem. Soc., 2017, 139, 15316; (c) C. Margarita and P. G. Andersson, J. Am. Chem. Soc., 2017, 139, 1346; (d) C. H. Basch, K. M. Cobb and M. P. Watson, Org. Lett., 2016, 18, 136; (e) H. M. Nelson, B. D. Williams, J. Miró and F. D. Toste, J. Am. Chem. Soc., 2015, 137, 3213; (f) C. Sun,
B. Potter and J. P. Morken, J. Am. Chem. Soc., 2014, 136, 6534; $(g)$ X. Feng, H. Jeon and J. Yun, Angew. Chem., Int. Ed., 2013, 52, 3989; (h) A. Guzman-Martinez and A. H. Hoveyda, J. Am. Chem. Soc., 2010, 132, 10634.

9 D. R. Edwards, Y. B. Hleba, C. J. Lata, L. A. Calhoun and C. M. Crudden, Angew. Chem., Int. Ed., 2007, 46, 7799.

10 Y. H. Zhang, C. F. Xu, J. F. Li and C. Y. Yuan, Chin. J. Chem., 2003, 21, 883.

11 S. A. Westcott, H. P. Blom and T. B. Marder, Inorg. Chem., 1993, 32, 2175.

12 Our recent studies on rhodium-catalyzed CAHB of functionalized alkene substrates focus on using the simple and readily available TADDOL- and BINOL-derived chiral phosphites and phosphoramidites. Our studies consistently find that these two privileged ligand scaffolds tend to be complementary in their effectiveness; in part, the choice of TADDOL- or BINOL-derived ligands hinges on the alkene substitution pattern.

13 The parent BINOL-derived ligand $\mathbf{B} 2$ gives a similar yield and regioselectivity but lower enantioselectivity for $(S)$-9a (90:10 er); see the ESI† for ligand screening data.

14 (a) V. M. Shoba and J. M. Takacs, J. Am. Chem. Soc., 2017, 139, 5740; (b) N. G. Leonard and P. J. Chirik, ACS Catal., 2018, 8, 342 ; see also ref. $8 b$.

15 The regioisomeric $\gamma$ - and $\beta$-boronic esters 6 and 7 often proved difficult to separate by chromatography on silica gel. Thus, the 6:7 ratio is determined via 31P NMR analysis of crude reaction mixture. The isolated yield of the major $\gamma$-regioisomer and its er are obtained after oxidation.

16 It is worth noting that unlike our previous work on 1,1disubstituted alkenes (ref. $5 a$ ) in which ortho-substitution of the arene leads to a change in regioselectivity, substrates bearing 2-substituted arenes in this series of 1,2-disubstituted internal vinylarenes do not significantly impact the regioselectivity. For example, in addition to $\mathbf{5 h}$, the 2-methylphenyl derived substrate $\mathbf{5}(\mathrm{Ar}=2$ methylphenyl) also undergoes regioselective $\gamma$-boration (13:1 rr) to afford 6 (Ar = 2-methylphenyl) but with a relatively modest level of enantioselectivity determined after oxidation $(74 \%, 91: 9 \mathrm{er})$; see the ESI. $\dagger$

17 V. B. Birman and X. Li, Org. Lett., 2006, 8, 1351.

18 For examples of stereoretentive cross-coupling of chiral secondary organoboron derivatives, see ref. $5 c, e$ and $(a)$ T. P. Blaisdell and J. P. Morken, J. Am. Chem. Soc., 2015, 137, 8712; (b) M. Daini and M. Suginome, J. Am. Chem. Soc., 2011, 133, 475.

19 For examples of stereoinvertive cross-coupling of chiral secondary organoboron derivatives, see: $(a)$ J. C. H. Lee, H.-Y. Sun and D. G. Hall, J. Org. Chem., 2015, 80, 7134; (b) L. Li, S. Zhao, A. Joshi-Pangu, M. Diane and M. R. Biscoe, J. Am. Chem. Soc., 2014, 136, 14027; (c) J. C. Lee, R. McDonald and D. G. Hall, Nat. Chem., 2011, 3, 894; (d) T. Awano, T. Ohmura and M. Suginome, J. Am. Chem. Soc., 2011, 133, 20738; (e) D. L. Sandrock, L. Jean-Gerard, C. Chen, S. D. Dreher and G. A. Molander, J. Am. Chem. 
Soc., 2010, 132, 17108; $(f)$ T. Ohmura, T. Awano and M. Suginome, J. Am. Chem. Soc., 2010, 132, 13191.

20 For ligand-controlled stereoretentive and stereoinvertive cross-coupling of chiral secondary organoboron derivatives, see: S. Zhao, T. Gensch, B. Murray, Z. L. Niemeyer, M. S. Sigman and M. R. Biscoe, Science, 2018, 362, 670 .

21 For a perspective on enantiospecific and iterative SuzukiMiyaura cross-couplings, see: J. P. G. Rygus and C. M. Crudden, J. Am. Chem. Soc., 2017, 139, 18124.

22 D. Imao, B. W. Glasspoole, V. S. Laberge and C. M. Crudden, J. Am. Chem. Soc., 2009, 131, 5024.
23 A. Ros and V. K. Aggarwal, Angew. Chem., Int. Ed., 2009, 48, 6289.

24 The 3,3'-bisphenyl substituents in ligand B1 play a significant role in determining regioselectivity. B2, the analogous BINOL-derived phosphoramidite derived from the parent BIONOL ring system, gives a slight preference for $\beta$-boration (ca. 2:1 rr) with (E)-14. Using B2, $\gamma, \delta$ unsaturated amides afford $\delta$-borated benzylic boronic esters in excellent yields and enantioselectivities; see: ref. $7 a$.

25 J. Lu, B. Pattengale, Q. Liu, S. Yang, W. Shi, S. Li, J. Huang and J. Zhang, J. Am. Chem. Soc., 2018, 140, 13719. 